\title{
A NEW INTEGRABLE SYSTEM ON THE SPHERE
}

\author{
Holger R. Dullin and Vladimir S. Matveev
}

\begin{abstract}
We present a new Liouville-integrable natural Hamiltonian system on the (cotangent bundle of the) sphere $S^{2}$. The second integral is cubic in the momenta.
\end{abstract}

\section{Introduction}

A Hamiltonian system is called natural if its Hamiltonian is the sum of a positive-definite kinetic energy and a potential. Natural Hamiltonian systems on (cotangent bundles of) closed surfaces admitting integrals polynomial in momenta are interesting for a number of reasons:

1. They are classical: The formulation of the problem is due at least to Darboux [7].

2. If a natural Hamiltonian system admits a real-analytic integral, then it admits an integral polynomial in momenta [21].

3. In particular, all known natural integrable Hamiltonian systems on surfaces have integrals polynomial in momenta.

4. The existence of an integral polynomial in momenta of degree one or two has a clear geometric background: the existence of an integral of degree one implies the existence of a one-parametric family of symmetries of the system. The existence of an integral of degree two implies the existence of so-called separating variables.

Natural Hamiltonian systems on closed surfaces that admit a nontrivial integral which is polynomial in momenta of degree one or two are completely understood: there exists a complete description and classification, see, for example, [5]. Up to now, only one family of natural Hamiltonian systems on a closed surface admitting an integral of degree three and admitting no (nontrivial) integral of degree one or two is explicitly known, namely the family of systems obtained from the Goryachev-Chaplygin top. In the next section we present a second, distinct, family of natural Hamiltonian systems on $T^{*} S^{2}$ with an integrable cubic in the momenta.

Knowing this new system suggests the following ways of further investigation:

1. Construction of bifurcation diagram and description of the topological structure of the system.

Received May 10, 2004.

2000 Mathematics Subject Classification. 37J35, 58F07, 58F17, 70H06, 70E40. 
2. Finding action-angle variables and explicit formulae for orbits.

3. Quantisation of the system.

4. Painlevé analysis.

We constructed this new system using methods based on an observation from [9]. We will describe the methods and how we applied them elsewhere. It seems that it is the only new integrable system with cubic integral on a closed manifold containing elementary functions only that can be found using these methods. But still one can construct many new local examples of metrics with integrable geodesic flows, which we will do in a joint paper with Tabov and Topalov.

A special class of natural Hamiltonian systems are geodesic flows, i.e., natural Hamiltonian systems with zero potential. According to Maupertuis's principle, an integrable natural Hamiltonian system immediately gives a family of integrable geodesic flows, see [3] for details. If the integral of the system is polynomial in momenta, the integrals of the geodesic flows are also polynomial of the same degree.

The investigation of geodesic flows that admit an integral polynomial in momenta is also a classical subject. If the degree of integrals is one or two, they were well-understood already in 19th century, see for example [7]. There are a lot of local examples of geodesic flows admitting integrals polynomial in velocities of degree 3 , see $[13,14,22]$ and the survey [12] for details. However, there are only very few global examples, i.e. on closed or complete surfaces, see below.

Let us recall the known results about geodesic flows on closed surfaces admitting an integral polynomial in momenta. For a more detailed review see [5].

First of all, in view of results of Kolokoltsov [18], a geodesic flow on a surfaces of genus greater than two cannot admit a nontrivial integral polynomial in momenta. Then, an orientable surfaces admitting such geodesic flows must be the sphere or the torus.

We collect the main results about existence and classification of metrics on the torus and on the sphere whose geodesic flow admits a nontrivial integral polynomial in momenta in the following table:

\begin{tabular}{|c|c|c|}
\hline & Sphere $S^{2}$ & Torus $T^{2}$ \\
\hline Degree 1 & All is known & All is known \\
\hline Degree 2 & All is known & All is known \\
\hline Degree 3 & Series of examples & Partial negative results \\
\hline Degree 4 & Series of examples & Partial negative results \\
\hline Degree $\geq 5$ & Nothing is known & Nothing is known \\
\hline
\end{tabular}

The following notation is used in the table. "Degree" means the smallest degree of a nontrivial integral polynomial in velocities. "All is known" means that there exists an effective description and classification (can be found in [4]). 
There exists only one explicit "Series of examples" for degree three. It comes from the Goryachev-Chaplygin case of rigid body motion (by applying symplectic reduction and Maupertuis's principle), see [3]. The most valuable "partial negative results" are due to Byalyi [6] and Denisova and Kozlov [8]. They proved that if a natural Hamiltonian system on the torus whose kinetic energy is given by a flat metric admits an integral polynomial in momenta of degree three, it admits an integral linear in momenta.

By applying Maupertuis's principle, our system gives one more series of examples of integrable geodesic flows on the sphere whose integral is polynomial in momenta of degree 3 . It is possible to show that these geodesic flows do not admit integrals which are polynomial in momenta of degree less than 3 , and that these examples are different from examples coming from Goryachev-Chaplygin. The proof is lengthy, and is given in the extended version [10] of this paper.

Recently Kiyohara [16] proved the existence of one more series of examples of integrable geodesic flows on the sphere whose integral is polynomial in momenta of degree 3. Although his examples are not explicit (similar to Selivanova [20] he writes a nonlinear differential equation and claims that its solutions give integrable geodesic flows), it is easy to see that his examples are different from ours, since they have regions of constant curvature and therefore cannot be realanalytic.

1.1. The result. Consider the sphere $S^{2} \subset \mathbb{R}^{3}$ of radius 1 with the spherical coordinates $(x, y, z)=(-\sin \theta \cos \phi,-\sin \theta \sin \phi, \cos \theta)$ and the following two functions $H$ (the Hamiltonian) and $F$ (the second integral) on the cotangent bundle of the sphere without poles $z= \pm 1$. Let $A, c, s \in \mathbb{R}$ be parameters with $s>1$ and define

$$
W(z)=z+s, \quad P(z)=3 z^{2}+4 s z+1, \quad Q(z)=3 z^{2}+2 s z-1
$$

and

$$
G(z)=\frac{P(z)}{(2 W(z))^{2}} .
$$

Then, the function $H$ is given by $H:=K+V$, where

$$
\begin{aligned}
& K:=\frac{1}{2}\left(\frac{1}{\sin ^{2} \theta}+G(\cos \theta)\right) p_{\phi}^{2}+\frac{1}{2} p_{\theta}^{2} \\
& V:=A \frac{\sin \theta}{\sqrt{W(\cos \theta)}} \cos \phi+\frac{c}{W(\cos \theta)},
\end{aligned}
$$

and $F$ is defined as

$$
F:=2 H p_{\phi}-p_{\phi}^{3}+A \cos (\phi) \frac{Q(\cos \theta)}{\sqrt{W(\cos \theta)} \sin \theta} p_{\phi}+2 A \sin \phi \sqrt{W(\cos \theta)} p_{\theta} .
$$

Proposition: The functions $H$ and $F$ can be analytically continued to the cotangent bundle of the whole sphere. The continuation is also polynomial in momenta (of degree 2 for $H$ and of degree 3 for $F$ ). 
We will denote the continuations of $H, K, F$ by the same letters $H, K, F$; in particular in the theorem below we mean the continued functions defined on the cotangent bundle of the whole sphere.

Theorem: The following statements hold:

1. The functions $H$ and $F$ commute with respect to the standard Poisson bracket on $T^{*} S^{2}$ and are functionally independent

2. The kinetic energy $K$ is positive definite

3. If $A \neq 0$, the Hamiltonian $H$ does not admit a (smooth) nontrivial integral which is polynomial in velocities of degree less than three and which is linearly independent of $H$.

In other words, we found a new 1) Liouville integrable, 2) natural Hamiltonian system on the sphere with 3) an integral cubic in momenta.

1.2. Why the system is new. The previously known family comes from the Goryachev-Chaplygin top using symplectic reduction, see [3] for the details. It is a natural Hamiltonian system on the sphere. In the standard spherical coordinates its Hamiltonian $H_{1}$ is $K_{1}+V_{1}$, where the kinetic energy $K_{1}$ and the potential $V_{1}$ are

$$
\begin{aligned}
K_{1} & :=\frac{1}{2}\left(\frac{\cos ^{2} \theta}{\sin ^{2} \theta}+4\right) p_{\phi}^{2}+\frac{1}{2} p_{\theta}^{2} \\
V_{1} & :=A_{1} \sin \theta \sin \phi,
\end{aligned}
$$

see, for example, [9]. The integral $F_{1}$ for the Hamiltonian $H_{1}$ is given by

$$
F_{1}:=H_{1} p_{\phi}-2 p_{\phi}^{3}-\frac{A_{1}}{2} p_{\theta} \cos (\phi) \cos (\theta)+\frac{A_{1}}{2} \frac{\sin \phi\left(3(\cos \theta)^{2}-2\right)}{\sin \theta} p_{\phi} .
$$

It is easy to see that the system we found is essentially different from the Goryachev-Chaplygin system. In order to show this, we will sketch the proof that there exists no diffemorphism $D: S^{2} \rightarrow S^{2}$ such that $D_{*} K=\alpha K_{1}$.

Consider the metrics $g, g_{1}$ corresponding to the kinetic energies $K, K_{1}$, respectively. They are given by the formulae:

$$
\begin{aligned}
d s^{2} & =d \theta^{2}+\frac{d \phi^{2}}{1 / \sin ^{2} \theta+G(\cos \theta)} \\
d s_{1}^{2} & =d \theta^{2}+\frac{d \phi^{2}}{4+\cot ^{2} \theta}=d \theta^{2}+\frac{d \phi^{2}}{1 / \sin ^{2} \theta+3} .
\end{aligned}
$$

The sketch of the proof is as follows (the proof can be found in [10]). The diffeomorphism $D$, if it exists, must take the lines of constant curvature $\theta=$ const, and hence also the orthogonal line $\phi=$ const into each other. Since $\phi$ is periodic, up to a shift of $\phi$ the map $D$ therefore only changes $\theta$, and then, by comparing formulas, it is easy to see that $d s^{2}$ and $d s_{1}^{2}$ cannot be mapped into each other by such a $D$. 
Selivanova [20] proved the existence of an additional family of natural Hamiltonian systems admitting integrals of degree 3 in momenta. This family is not explicit. Instead, a nonlinear differential equation is derived and it is proved that certain solutions of this equation allow to construct a geodesic flow admitting an integral of degree three and admitting no integral of smaller degree. We believe that our explicit system does not overlap with the implicit system of Selivanova.

\section{Proof of the proposition}

The second integral $F$ is cubic in the momenta, like the Goryachev-Chaplygin top. This top does not appear as a limiting case in our family, but is serves as a motivation for the following coordinate transformation, which uses global coordinates $(x, y, z)$ on the unit sphere embedded in $\mathbb{R}^{3}$. The angular momentum $\left(L_{x}, L_{y}, L_{z}\right)$ where $L_{z}=x p_{y}-y p_{x}=p_{\phi}$ etc. with cyclic permutations, satisfies the usual Poisson structure on the sphere with non-vanishing brackets

$$
\left\{L_{x}, L_{y}\right\}=L_{z}, \quad\left\{x, L_{y}\right\}=z,\left\{z, L_{y}\right\}=-x
$$

and cyclic permutations thereof. This bracket has Casimirs $x^{2}+y^{2}+z^{2}=1$ and $x L_{x}+y L_{y}+z L_{z}=0$. The global Hamiltonian is $H=K+V$, where

$$
\begin{aligned}
& K=\frac{1}{2}\left(L_{x}^{2}+L_{y}^{2}+(1+G(z)) L_{z}^{2}\right) \\
& V(x, y, z)=-A \frac{x}{\sqrt{W(z)}}+\frac{c}{W(z)},
\end{aligned}
$$

and is clearly real-analytic. The integral reads

$$
\begin{aligned}
F & =2 H L_{z}-L_{z}^{3}-A \frac{Q}{\sqrt{W}} \frac{x L_{z}}{1-z^{2}}-2 A \sqrt{W} \frac{y\left(x L_{y}-y L_{x}\right)}{1-z^{2}} \\
& =2 H L_{z}-L_{z}^{3}+\frac{A}{\sqrt{W}}\left(x L_{z}+2 W L_{x}\right) .
\end{aligned}
$$

In the second line the apparent singularity in $F$ is removed by using both Casimirs $x^{2}+y^{2}+z^{2}=1$ and $x L_{x}+y L_{y}+z L_{z}=0$ and in addition the identity $Q=2 z W+z^{2}-1$. The integral is cubic in the momenta, and clearly is real-analytic.

Like the Goryachev-Chaplygin top the system is only integrable on the level set of the Casimir $x L_{x}+y L_{y}+z L_{z}=0$. Writing the Hamiltonian in this form suggests an interpretation of a spinning top with zero angular momentum (the vanishing Casimir), an orientation dependent moment of inertia, and a nonstandard potential. Note, however, that $1+G$ is not positive for all $z \in[-1,1]$ for certain values of $s$, which means that a moment of inertia may pass through infinity for such value of $s$. Nevertheless, the kinetic energy in $H$ is positive definite for all $s$, see Section 3.2. This is possible even when $1+G<0$ because $x L_{x}+y L_{y}+z L_{z}=0$. 


\section{Proof of the Theorem}

3.1. Liouville integrable. It is tedious but easy to check that the functions $H$ and $F$ commute, since they are given by explicit formulae. The calculation goes as follows. The canonical bracket is a quadratic polynomial in the momenta. The term independent of the momenta vanishes because $Q=2 z W-1+z^{2}$. The coefficient of $p_{\theta} p_{\phi}$ vanishes because $P=4 z W+1-z^{2}$. The nontrivial fact is that the coefficients of $p_{\phi}^{2}$ which contains $P, Q$ and derivatives thereof vanishes. A similar (but harder) calculation can be done using the global coordinates of the previous section.

$H$ and $F$ are independent because $H$ has terms quadratic in momenta (and not a square of a linear function of momenta) and $F$ has terms that are cubic in momenta (and not a square of a linear function of momenta). So $H$ and $F$ have vanishing Poisson bracket and are functionally independent, hence the system is Liouville integrable.

3.2. Natural. By introducing local coordinates near the poles $z= \pm 1$ it is easy to see that the kinetic energy $K$ is positive definite near these points, see [10] for detailed calculations. Thus, it is sufficient to show that the metric (1) is positive definite at regular points of the spherical coordinate systems, i.e. we have to show that $C:=1 / \sin ^{2} \theta+G(\cos \theta)$ is positive for $0<\theta<\pi$. Substituting $z$ instead of $\cos \theta$, we obtain

$$
C(z)=\frac{1}{4} \frac{6 z^{2}+12 s z+4 s^{2}-3 z^{4}-4 s z^{3}-1}{\left(1-z^{2}\right)(z+s)^{2}} .
$$

Since the denominator $\left(1-z^{2}\right)(z+s)^{2}$ is always positive for $-1<z=\cos (\theta)<$ 1 , we need to prove that the numerator

$$
R(z):=\frac{3}{2} z^{2}+3 s z+s^{2}-\frac{3}{4} z^{4}-s z^{3}-\frac{1}{4}
$$

is positive. Its value at $z=-1$ is $(s-1)^{2}$ and hence positive; its derivative is

$$
\frac{\partial R(z)}{\partial z}=3\left(1-z^{2}\right)(z+s) \text {. }
$$

Since the derivative is also positive for $-1<z=\cos (\theta)<1, R(z)$ is positive for $-1<z=\cos (\theta)<1$. Hence the metric (1) is positive definite.

3.3. Cubic. Now let us prove that, for $A \neq 0$, our system does not admit an integral linear in momenta, and that every integral quadratic in momenta is proportional to the Hamiltonian. An analytic proof of this statement can be found in [10], here we give a geometric proof.

Clearly, if $A \neq 0$, the integral $F$ is not the third power of a function linear in momenta, and is not the product of the Hamiltonian and a function linear in momenta. Hence, the existence of an integral which is linear or quadratic in momenta implies that the system is resonant: every orbit is a closed curve.

But this could not be true. Indeed, take a sufficiently big $h \in \mathbb{R}$, and apply Maupertuis's principle to the energy level $h$. We obtain a metric on $S^{2}$ such that 
its (unparameterised) geodesics are projections of the orbits of our system lying on the energy level $h$. If all orbits are closed curves, all geodesics are closed curves as well. The theory of metrics all whose geodesics are closed is welldeveloped [1]. It is known [11] that on the two-sphere geodesics of such metrics have no self-intersections. But if $h$ is very big, certain geodesics of our metric must have self-intersections: indeed, for huge $h$, the geodesics of our metric are very close to the geodesic of the metric (1), and by direct analysis it is possible to see that certain geodesics of (1) do have self-intersections.

Thus, our system has no integral of degree one or two. This proves the Theorem.

\section{Remark}

After this paper was accepted we became aware of a paper by Yehia [22], in which the system in local coordinates was derived. However, the essential observation that it is in fact a smooth natural system on the sphere is new.

\section{Acknowledgments}

The authors thank H. Yehia for his comments. VSM thanks the DFGprogramm 1154 (Global Differential Geometry) and Ministerium für Wissenschaft, Forschung und Kunst Baden-Württemberg (Eliteförderprogramm Postdocs 2003) for partial financial support. HRD thanks the Centre Bernoulli EPF Lausanne for the hospitality in Summer 2004 when this paper was finished.

\section{References}

[1] A. L. Besse, Manifolds all of whose geodesics are closed, Springer-Verlag 1978.

[2] G. D. Birkhoff, Dynamical Systems, A.M.S. Colloq. Publ. 9, Amer. Math. Soc., New York, 1927.

[3] A. V. Bolsinov, V. V. Kozlov, A. T. Fomenko, The Maupertuis's principle and geodesic flows on $S^{2}$ arising from integrable cases in the dynamics of rigid body motion, Russ. Math. Surv. 50 (1995) 473-501.

[4] A. V. Bolsinov, V. S. Matveev, A. T. Fomenko, Two-dimensional Riemannian metrics with an integrable geodesic flow. Local and global geometries, Sb. Math. 189 (1998), 1441-1466.

[5] A.V. Bolsinov, A. T. Fomenko, Integrable geodesic flows on two-dimensional surfaces, Monographs in Contemporary Mathematics. Consultants Bureau, New York, 2000.

[6] M. L. Byalyĭ, First integrals that are polynomial in the momenta for a mechanical system on the two-dimensional torus, Functional Anal. Appl. 21 (1987), 310 - 312.

[7] G. Darboux, Lecons sur la théorie générale des surfaces, Vol. 3, Chelsea Publishing 1894.

[8] N. V. Denisova, V. V. Kozlov, Polynomial integrals of reversible mechanical systems with a two-dimensional torus as configuration space, Sb. Math. 191 (2000), 43-63.

[9] H. R. Dullin, V. S. Matveev, P. J. Topalov, On the integrals of third degree in momenta, Regul. Chaot. Dyn. 4 (1999), 35-44.

[10] H. R. Dullin, Vladimir S. Matveev, A new integrable system on the sphere, preprint, http://xxx.lanl.gov/abs/math.DS/0406209

[11] D. Gromoll, K. Grove, On metrics on $S^{2}$ all of whose geodesics are closed, Invent. Math. 65 (1981), 175-177. 
[12] J. Hijetarinta, Direct methods for the search of the second invariant, Phys. Rep. 147(1987) no. 2, 87-154.

[13] M. Karlovini, K. Rosquist, A unified treatment of cubic invariants at fixed and arbitrary energy, J. Math. Phys. 41(2000), no. 1, 370-384.

[14] M. Karlovini, G. Pucacco, K. Rosquist, L. Samuelsson, A unified treatment of quartic invariants at fixed and arbitrary energy, J. Math. Phys. 43(2002), no. 8, 4041-4059.

[15] K. Kiyohara, Compact Liouville surfaces, J. Math. Soc. Japan 43(1991), 555-591.

[16] K. Kiyohara, Two-dimensional geodesic flows having first integrals of higher degree, Math. Ann. 320(2001) no 3, 487-505.

[17] V. N. Kolokol'tzov, Polynomial integrals of geodesic flows on compact surfaces, Dissertation, Moscow State University 1984.

[18] V. N. Kolokol'tzov, Geodesic flows on two-dimensional manifolds with an additional first integral that is polynomial with respect to velocities, Math. USSR-Izv. 21(1983), no. 2, 291-306.

[19] O. E. Orel, Rotation function for integrable problems that reduce to Abel equations. Orbital and classification of Goryachev-Chaplygin system, Sb. Math. 186(1995) no. 2, 271-296.

[20] E. N. Selivanova, New examples of Integrable Conservative Systems on $S^{2}$ and the Case of Goryachev-Chaplygin, Comm. Math. Phys. 207(1999) 641-663.

[21] E. T. Whittaker, A treatrise on the Analytical Dynamics of Particles and Rigid Bodies, Cambridge University Press 1937.

[22] H. M. Yehia, On certain two-dimensional conservative mechanical systems with a cubic second integral, J. Phys. A 35 (2002) 9469-9487

Department of Mathematical Sciences, Loughborough University, LE11 3TU UK

E-mail address: h.r.dullin@lboro.ac.uk

Mathematisches Institut, Universität Freiburg, 79104 Germany

E-mail address: matveev@email.mathematik.uni-freiburg.de 